\title{
Experimental Evaluation of Shore to Unmanned Surface Vehicle Wi-Fi Communications
}

\author{
André Coelho, Mário Lopes, Bruno Ferreira, Rui Campos, Manuel Ricardo \\ INESC TEC and Faculdade de Engenharia, Universidade do Porto \\ Rua Dr. Roberto Frias, 378 \\ 4200-465 Porto, Portugal \\ \{afcoelho, mjlopes, bmmf, rcampos, mricardo\}@inesctec.pt
}

\begin{abstract}
The blue economy potential is envisioned to increase the activity at the ocean worldwide in the coming years. To support these activities and the convergence to the Internet of Moving Things, Unmanned Surface Vehicles (USVs) are considered viable platforms to enable a large number of missions, including border surveillance and environmental monitoring. Typically, USVs use Wi-Fi for communicating with shore. However, in the literature, there is a lack of studies characterizing the shore-to-USV WiFi link. This paper studies the influence of distance and USV orientation on the shore-to-USV link quality at the $2.4 \mathrm{GHz}$ and 5.8 GHz Industrial, Scientific, and Medical (ISM) bands. The study is supported by experimental results, collected during sea trials. For the $2.4 \mathrm{GHz}$ band, we conclude that neither the TwoRay propagation model nor the Friis propagation model allow a good fit to the experimental measurements. On the other hand, for the $5 \mathrm{GHz}$ band, the Friis propagation model fits the obtained experimental results.
\end{abstract}

Index Terms-Experimental evaluation, Unmanned Surface Vehicle, Wi-Fi communications, propagation models.

\section{INTRODUCTION}

The blue economy potential is envisioned to increase the activity at the ocean worldwide in the coming years, including resource exploitation on the sea floor, environmental monitoring, scientific exploration, and surveillance of maritime borders, apart from the traditional activities such as maritime transportation and fisheries [1]. To support these activities and the convergence to the Internet of Moving Things, Unmanned Surface Vehicles (USVs) are considered viable platforms to enable a large number of missions, including border surveillance and environmental monitoring.

Due to the small size of this type of vehicles and their proximity to the sea surface, there are many factors that may affect the performance of wireless communications between a USV and an on shore Base Station (BS). Firstly, the reflection effect on the sea surface and the scattering phenomenon caused by the roughness of the sea waves result in multipath components of the transmitted signal [2]. Additionally, the movements of the USVs, due to the sea waves, result in changes in the angle between the transmitting and receiving antennas [3]. Finally, the effect of the Earth curvature can limit the radio propagation due to the obstruction of the Lineof-Sight (LoS). All these phenomena may contribute to the degradation of the received signal strength [3].

This paper evaluates the variation of the Wi-Fi link quality at $2.4 \mathrm{GHz}$ and $5.8 \mathrm{GHz}$ between a USV at sea and a BS located on shore. In particular, the influence of distance and the USV orientation with respect to the BS on shore is studied. Moreover, experimental results are compared with the two state of the art propagation models usually considered for over sea communications, the Two-Ray and Friis propagation models.

The rest of the paper is organized as follows. Section II describes the related work. Section III details the experimental setup and the methodology used to achieve the experimental results. The experimental results are presented and analyzed in Section IV. Finally, Section V points out the main conclusions and the future work.

\section{RELATED WORK}

Wireless links are commonly used to enable communications between USVs and shore stations. Examples are the USV for water quality monitoring presented in [4], which uses a $915 \mathrm{MHz}$ link for telemetry and Zigbee, operating at $2.4 \mathrm{GHz}$, to exchange sensors data, the Zarco [5] and the Unmanned Capsule (UCAP) [6] solutions, developed by INESC TEC, which use Wi-Fi as communications technology, and the prototypes designed in [7], wherein Wi-Fi and WiMAX are proposed to achieve long-range links. However, in the literature, to the best of our knowledge, there is a lack of studies characterizing shore-to-USV wireless link, especially for small USVs. In the following, the most relevant related works using other types of surface vehicles are identified.

In [8], a channel characterization, including path-loss, channel correlation, Doppler effects, and sea reflections, affecting $2 \mathrm{GHz}$ radio propagation over sea, for a land-to-ship channel with spatial diversity, is presented. It was concluded that a fading component was introduced by the reflections on the sea surface. In [9], a radio propagation study focused on sea environments is performed. The authors concluded that the characterization of channels above sea depends strongly on the environment and antenna heights. Additionally, they concluded the correlation between the experimental results and some theoretical models. Shore-ship LoS radio propagation at $5 \mathrm{GHz}$ was studied in [10]. The authors measured the received signal strength for a distance up to $10 \mathrm{~km}$ and compared the experimental results with the Two-Ray and Friis propagation models. They concluded that the measured results were close to the ones obtained using the Two-Ray model. However, 
when the distance increases, the authors report the influence of evaporating ducts that affect the prediction achieved using the Two-Ray model. In [3], a novel radio propagation model taking into account the effects of ship motions and the Earth curvature is designed. By applying this propagation model, the authors concluded that the impact of ship motions on radio propagation becomes smaller with the increase of transmission distance, and it is more significant at higher frequencies. In [11], experimental propagation measurements for $5.8 \mathrm{GHz}$, which were performed in a sea port, are presented. The study was focused on extracting time dispersion characteristics for radio channels, and it allowed to conclude that the $5.8 \mathrm{GHz}$ band is well suited for maritime communications. In [12], a performance evaluation of a $5.8 \mathrm{GHz}$ point-to-point link, between a fishing ship and shore BS is presented. Experimental results showed that links up to $7 \mathrm{~km}$ at $1 \mathrm{Mbps}$ are possible, showing the advantage of using long range IEEE 802.11 links for broadband maritime communications.

\section{EXPERIMENTAL SETUP}

In this section, the setup used to achieve the experimental results is characterized, including the communications nodes used, the testbed created, and the variables collected along the experiments.

\section{A. Communications nodes}

The communications nodes consist of a Base Station (BS) and one USV developed in the context of the FLEXUS ${ }^{1}$ project [13]. The BS (Figure 1) is a static node that acts as a gateway to the Internet; the USV (Figure 2) is a catamaran with $0.90 \mathrm{~m}$ of length and $0.65 \mathrm{~m}$ of height; Both the USV and BS are equipped with two Network Interface Cards (NICs) operating at $2.4 \mathrm{GHz}$ and $5.8 \mathrm{GHz}$ bands, and four omnidirectional antennas, two for each band, as illustrated in Figure 2, in order to take advantage of the IEEE 802.11n spatial diversity technique. The BS antennas were fixed on a tripod and the USV antennas were fixed on the vehicle frame.

\section{B. Testbed}

The experiments were carried out in different periods between $6^{\text {th }}$ and $7^{\text {th }}$ September 2017, in the Gulf of Elefsina sea in Athens, Greece. The sea was flat most of the time. The BS was placed on shore and the USV was moved over the sea, following the trajectories depicted in Figure 3. The NICs physical data rate was configured to auto mode but switching only between Modulation and Coding Scheme (MCS) 0 and 1. The $2.4 \mathrm{GHz}$ NICs used a frequency channel with $40 \mathrm{MHz}$ bandwidth; the $5.8 \mathrm{GHz}$ NICs used a frequency channel with $20 \mathrm{MHz}$ bandwidth. The transmission power of both the USV and BS was set to $22 \mathrm{dBm}$ at $2.4 \mathrm{GHz}$ and $25 \mathrm{dBm}$ at $5.8 \mathrm{GHz}$. For the $2.4 \mathrm{GHz}$ band, the BS and USV $4 \mathrm{dBi}$ antennas were placed respectively at $5.83 \mathrm{~m}$ and $0.39 \mathrm{~m}$ above the sea level; for the $5.8 \mathrm{GHz}$ band, the BS and USV $3 \mathrm{dBi}$ antennas were placed respectively at $6.06 \mathrm{~m}$ and $0.55 \mathrm{~m}$. The antennas height above the sea level was restricted by the structures to which

\footnotetext{
${ }^{1}$ http://flexus.inesctec.pt
}

they were fixed. The antenna gains were only considered for the USV. For the BS antennas, by considering 1) their altitude above the sea level, 2) E-plane measurements, and 3) the USV operation range between $50 \mathrm{~m}$ and $450 \mathrm{~m}$, the following antenna gains were calculated. The $2.4 \mathrm{GHz}$ BS antennas gain was around $2.5 \mathrm{dBi}$ for all distances; on the other hand, the $5.8 \mathrm{GHz} \mathrm{BS}$ antennas gain was $-3.7 \mathrm{dBi}$ for distances around $50 \mathrm{~m},-2.2 \mathrm{dBi}$ for $60 \mathrm{~m}$ and $70 \mathrm{~m},-0.7 \mathrm{dBi}$ between $80 \mathrm{~m}$ and $110 \mathrm{~m}$, and $0.5 \mathrm{dBi}$ between $120 \mathrm{~m}$ and $450 \mathrm{~m}$. The dependency of the antenna gains with distance was taken into account to obtain the theoretical results presented in Section IV for the Two-ray and Friis propagation models.

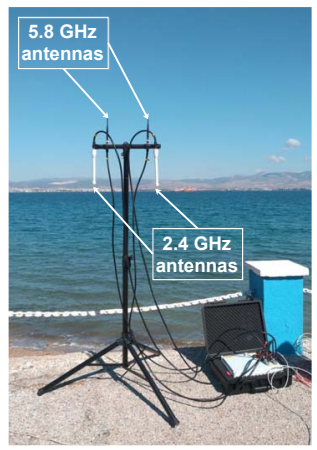

Fig. 1: The Base Station deployed on top of a $4 \mathrm{~m}$ high concrete platform.

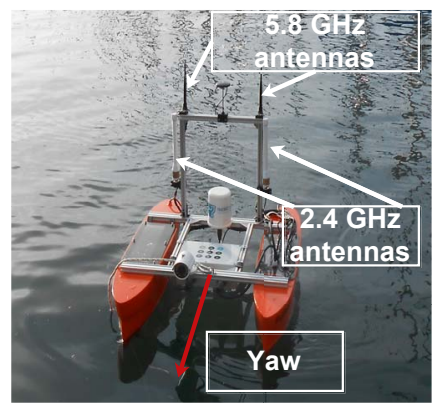

Fig. 2: The USV used in the sea trials.

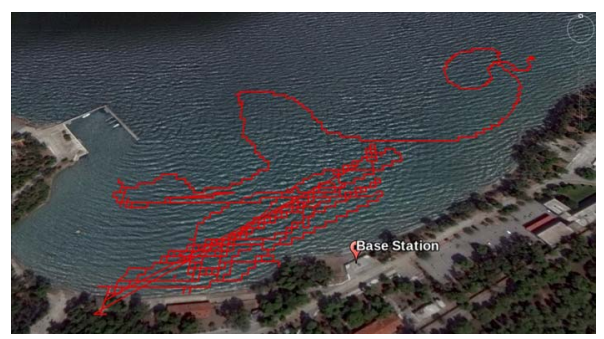

Fig. 3: The trajectories followed by the USV during the sea trials.

\section{Data collection}

The real conditions experienced by the USV throughout the experiments were collected periodically by means of three 
variables: 1) the quality of the radio links at $2.4 \mathrm{GHz}$ and $5.8 \mathrm{GHz}$; 2) the USV position over time; 3) the USV axis orientation. In the following, the way these variables were collected and their processing is explained.

- Quality of the radio link. This was achieved by collecting 1) the Received Signal Strength Indicator (RSSI) and 2) the Noise floor power, which are reported by the IEEE 802.11n NICs used on each node. Based on these variables, the Signal to Noise Ratio (SNR) for the link between the BS and the USV was calculated.

- USV position and distance to the BS. Using a GPS receiver on the USV, the $2 \mathrm{D}$ position coordinates (latitude and longitude) were collected periodically. Since the BS position was known during the experiments, the distance to the USV was calculated by applying the Haversine Formula [14].

- USV axis orientation. Since the USV is equipped with a magnetometer, the pitch, roll, and yaw were periodically collected. Taking into account USV and BS coordinates, USV heading to the BS was calculated based on the USV yaw values, considering the BS as reference, as depicted in Figure 4. Due to some incoherent experimental values for the pitch and roll axis, we do not include their influence in the study presented herein.

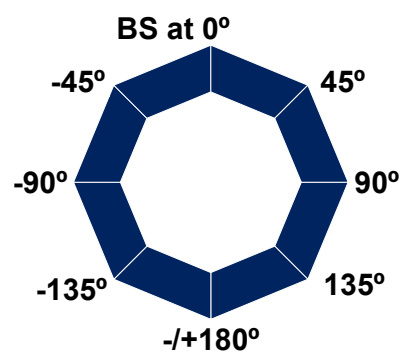

Fig. 4: Heading of the USV to the BS.

\section{EXPERIMENTAL RESULTS}

The obtained experimental results are presented in this section. The results are expressed in the form of average values with a $95 \%$ confidence interval and compared with the results obtained using the Two-ray and Friis propagation models.

\section{A. SNR versus Distance}

The variation of $\mathrm{SNR}$ at $2.4 \mathrm{GHz}$ and $5.8 \mathrm{GHz}$ versus the distance between the USV and the BS is depicted in Figures 5 and 6 . The SNR obtained using the Two-Ray and Friis propagation models is also shown, considering a constant Noise floor power of $-95 \mathrm{dBm}$, in order to be consistent with the noise power observed along the experiments. The results are divided into four segments, according to the USV heading to the BS, which represents the angle between the USV and BS antennas. This division aims at evaluating the real influence of distance on the radio link quality for approximately the same alignment between the USV and BS antennas. In the following, the most relevant conclusions for the $2.4 \mathrm{GHz}$ and $5.8 \mathrm{GHz}$ bands are presented.
1) $2.4 \mathrm{GHz}$ band: for distances up to $200 \mathrm{~m}$, a significant variation on the SNR values is observed, which may be justified by the USV antennas tilt variation to the BS. We believe the NICs Auto Gain Control (AGC), which instructs the Low Noise Amplifiers (LNAs) in the receiving radio chain to attenuate/amplify the signal, does not act with the required responsiveness. For the radio propagation over the sea, the Earth curvature needs to be taken into account, since it may become an obstruction to the Fresnel ellipsoid clearance and cause a degradation of the SNR, as mentioned in the literature [15]. In fact, as from $210 \mathrm{~m}$, the first Fresnel ellipsoid is obstructed up $40 \%$. This justifies the sudden decrease in the SNR from this distance on (cf. Figure 5). Overall, neither the Two-Ray propagation model nor the Friis propagation model provide a good fit to the experimental measurements, which can be justified by the used small size USV, navigating very close to the water, and by the placement of the $2.4 \mathrm{GHz}$ antennas of the USV, for which a side-wards obstruction to the radio propagation occurs. Although in some situations one USV antenna is blocked towards the BS antennas (Figures $5 \mathrm{~b}$ and $5 \mathrm{~d}$ - $1 \times 2$ system), the SNR values are almost the same when the two USV antennas are in LoS towards the BS antennas (Figures $5 \mathrm{a}$ and $5 \mathrm{c}-2 \times 2$ system). According to the definition of the maximum-ratio combining (MRC) technique [16], the SNR values are slightly lower in Figures $5 \mathrm{~b}$ and $5 \mathrm{~d}$ in relation to the ones in Figures $5 \mathrm{a}$ and $5 \mathrm{c}$.

2) $5.8 \mathrm{GHz}$ band: in general, the experimental results match the Friis propagation model, even though the TwoRay propagation model is also a good match for distances lower than $200 \mathrm{~m}$. For this frequency band, the clearance of the Fresnel ellipsoid was assured for the considered distances. In addition, since the $5.8 \mathrm{GHz}$ antennas were placed in the highest point of the USV mast, good radio propagation conditions were achieved. Typically, there is no obstruction between the USV antennas and the BS antennas; the exception occurs when the USV heading to the BS is $\pm 90^{\circ}$. Due to this fact, the SNR values are almost the same for all plots in Figure 6 .

\section{B. SNR versus Heading to the BS}

In Figure 7, the variation of $\mathrm{SNR}$ at $2.4 \mathrm{GHz}$ and $5.8 \mathrm{GHz}$ versus the USV heading to the BS is depicted. The results are divided into seven segments, considering the different distances between the USV and the BS.

By observing the plots of Figure 7, we can draw the following conclusions. Firstly, the $5.8 \mathrm{GHz}$ band is more stable than the $2.4 \mathrm{GHz}$ band, since the fluctuations between average values in each plot have lower amplitude, especially when the distance between the BS and the USV is lower than $200 \mathrm{~m}$. Additionally, the measured SNR for this band is, typically, lower than for the $2.4 \mathrm{GHz}$ band, namely when the distance is lower than $200 \mathrm{~m}$. For distances up to $200 \mathrm{~m}$, the decrease of the SNR for the $2.4 \mathrm{GHz}$ band can be justified by the LoS obstruction caused by the Earth curvature. In spite of such particularities, some general aspects can be identified for both bands. The first is the decreasing influence of the USV heading as distance increases, especially above $300 \mathrm{~m}$. Furthermore, the 


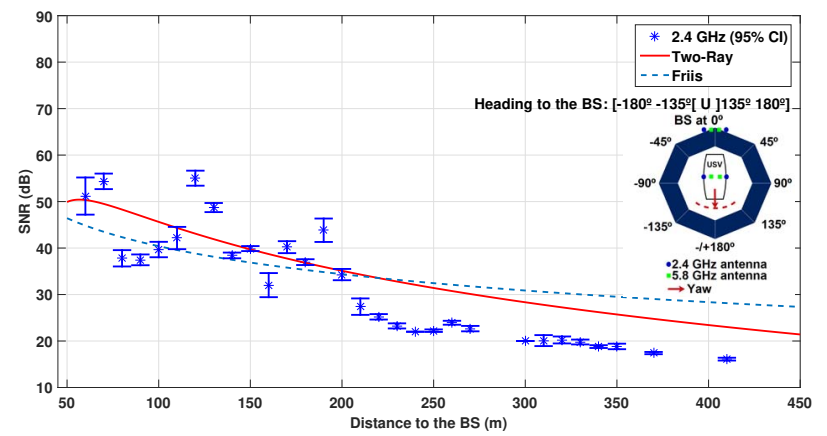

(a)

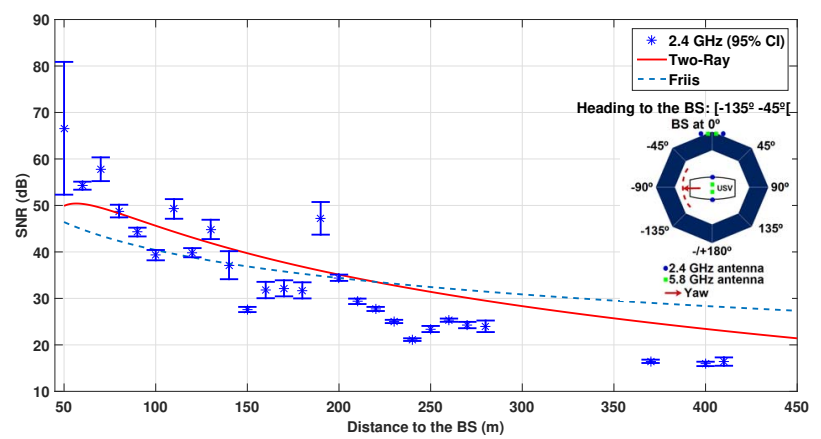

(b)

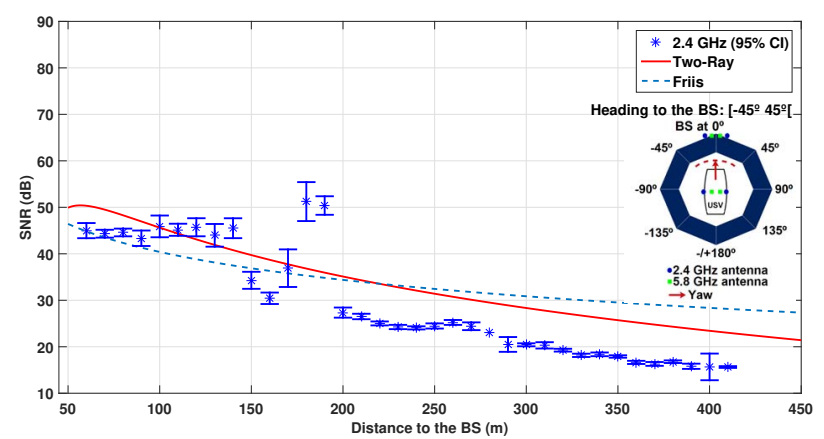

(c)

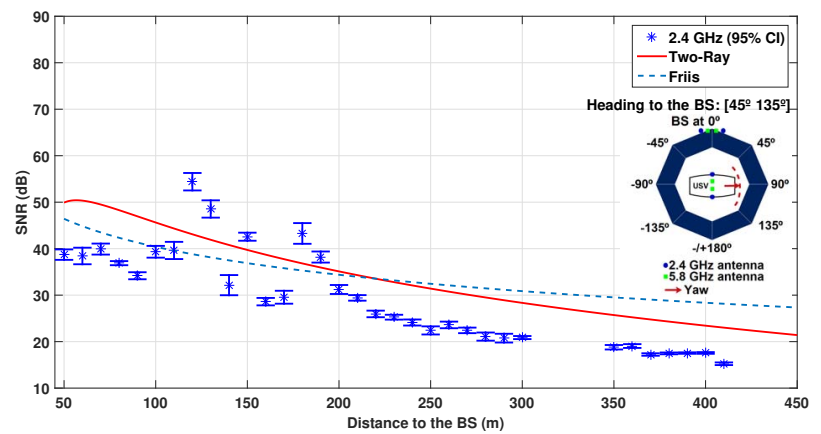

(d)

Fig. 5: SNR at $2.4 \mathrm{GHz}$ versus distance to the BS, from the USV point of view, against the Two-Ray and Friis propagation models.

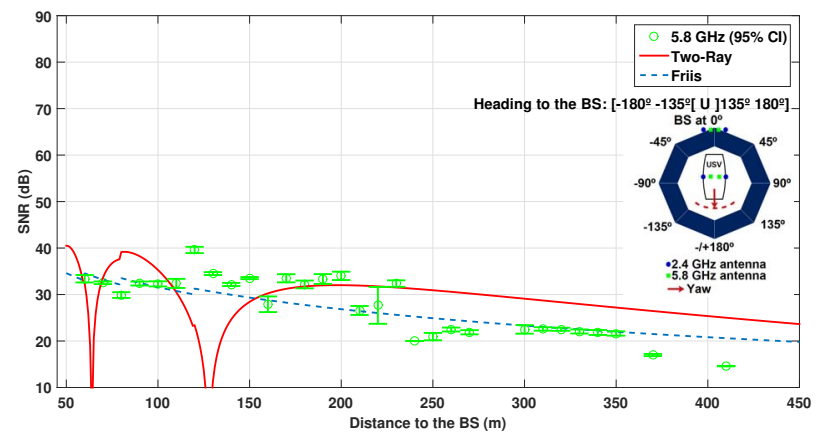

(a)

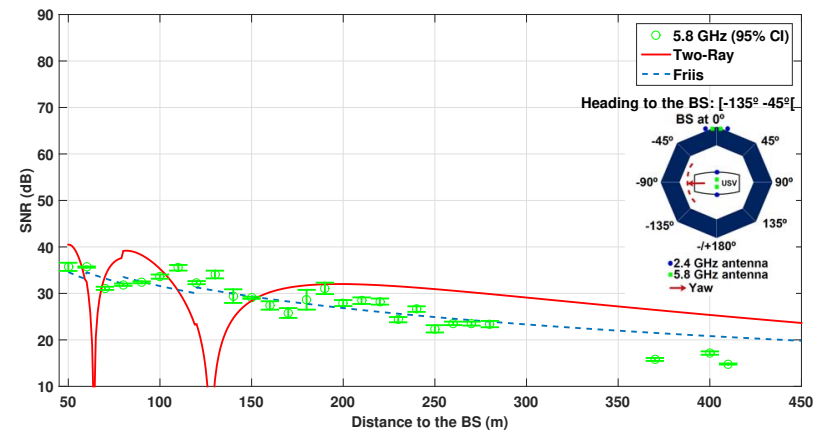

(b)

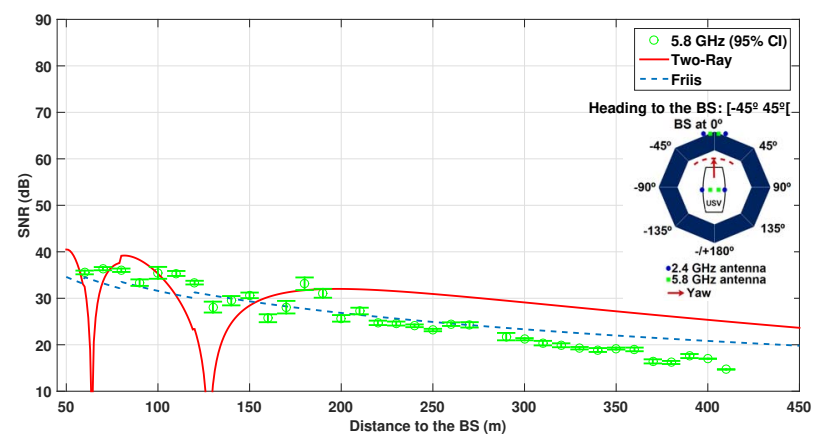

(c)

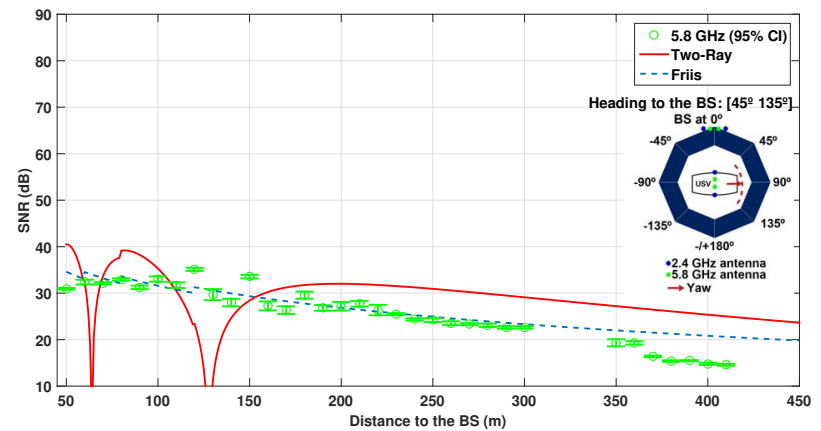

(d)

Fig. 6: SNR at $5.8 \mathrm{GHz}$ versus distance to the $\mathrm{BS}$, from the USV point of view, against the Two-Raw and Friis propagation models. 


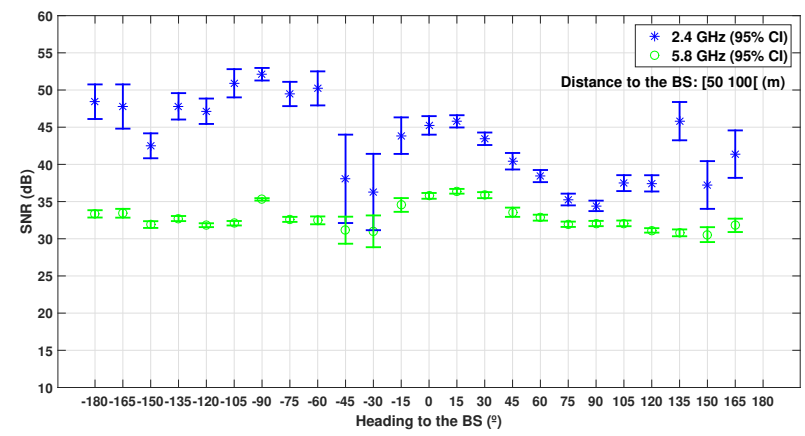

(a)

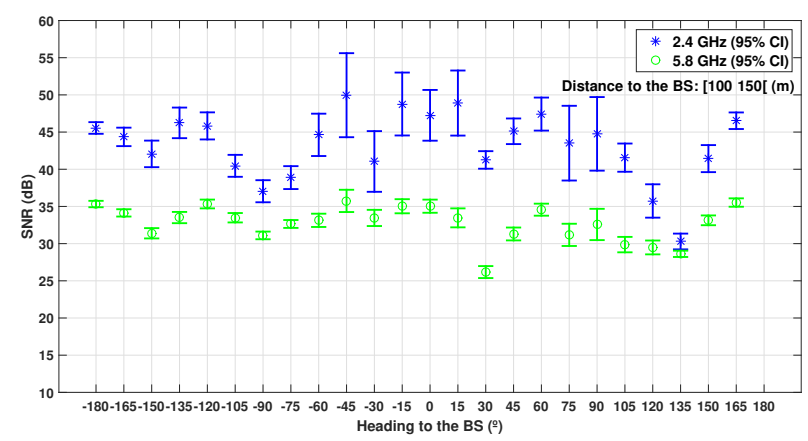

(b)

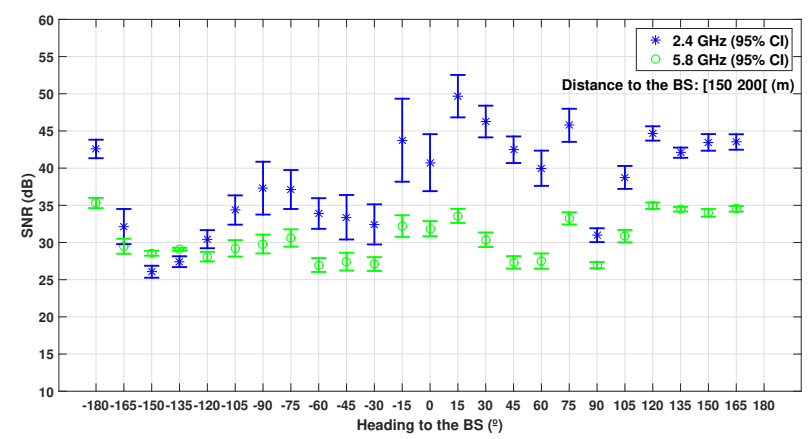

(c)

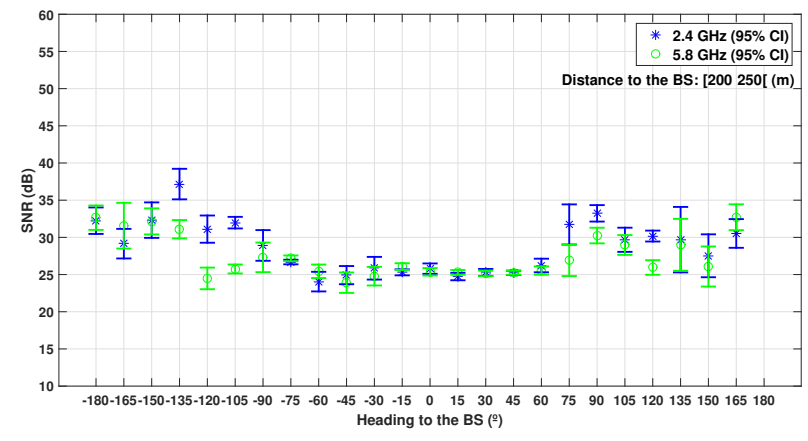

(d)

same trend is followed by both bands, regarding the increase and decrease of the average SNR under the same conditions. Globally, the highest SNR values are registered when the USV heading is close to $0^{\circ}$ or $\pm 180^{\circ}$, i.e., when both the USV and

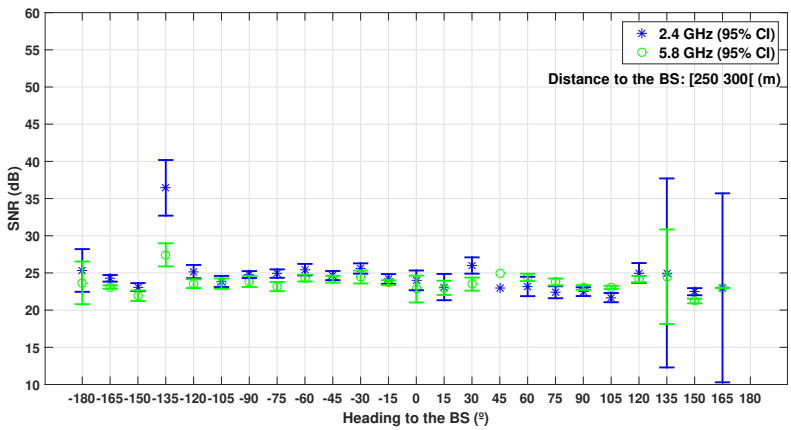

(e)

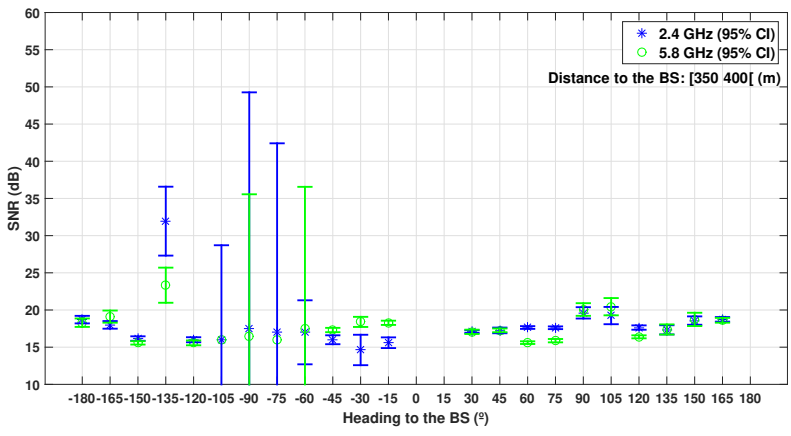

(f)

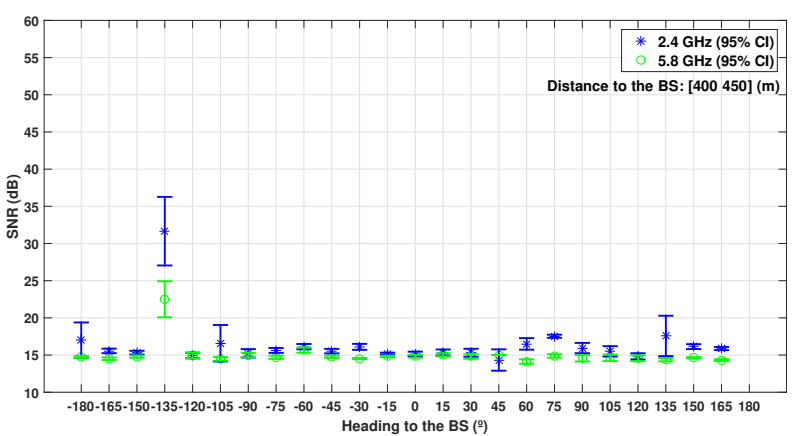

(g)

Fig. 7: SNR versus heading to the BS from the USV point of view.

BS antennas tend to be fully aligned. $2.4 \mathrm{GHz}$ SNR reduction when the heading of the USV to the BS is close to $\pm 90^{\circ}$ occurs mainly due to radio signal obstruction of one antenna, which implicitly disables spatial diversity.

\section{CONCLUSIONS}

In this paper, we evaluated the influence of distance and heading on the Wi-Fi link quality for the $2.4 \mathrm{GHz}$ and $5.8 \mathrm{GHz}$ bands, between a moving USV and a BS on shore. Considering the Two-Ray and Friis propagation models as baseline, we compared the theoretical predicted values for the SNR, with a set of experimental measurements collected during sea trials. For the $2.4 \mathrm{GHz}$ band, we concluded that neither the TwoRay propagation model nor the Friis propagation model allow a good fit to the experimental measurements; conversely, for 
the $5.8 \mathrm{GHz}$ band, the Friis propagation model matches the obtained experimental results. Additionally, we showed the possible negative effect of the Earth curvature on the $2.4 \mathrm{GHz}$ link quality for distances between the USV and the BS above $200 \mathrm{~m}$. This is due to the the small size USV navigating very close to the water. Regarding the heading of the USV to the $\mathrm{BS}$, we concluded that its influence on link quality is negligible for distances above $300 \mathrm{~m}$. On the other hand, for near-shore operations, the SNR will be higher when the heading tends to $0^{\circ}$ or $\pm 180^{\circ}$, i.e., when the USV and BS antennas tend to be perfectly aligned, as it would be expected. Lastly, we pointed out that the $2.4 \mathrm{GHz}$ band allows to achieve higher SNR values than the $5.8 \mathrm{GHz}$ band, for the same conditions, however the fluctuations on the experimental SNR values for the $5.8 \mathrm{GHz}$ band are smoother. As such, it may be more suitable to use the $5 \mathrm{GHz}$ band than the $2.4 \mathrm{GHz}$ band for shore-to-USV Wi-Fi communications.

As future work, it would be important to collect accurate pitch and roll data with another sensor, so we can better characterize the real impact of those variables on the radio link in both frequency bands. Additionally, a rearrangement of the placement for the USV antennas can be explored, namely putting the $2.4 \mathrm{GHz}$ antennas in the highest point of the USV mast. Furthermore, tests considering the use of IEEE 802.11n MIMO spatial multiplexing may be considered.

\section{ACKNOWLEDGEMENTS}

This work has been implemented in the context of the RAWFIE project. RAWFIE has received funding from the European Union Horizon 2020 Research and Innovation programme under grant agreement No. 645220.

This work is part of the project STRONGMAR: STRengthening MARritime technology Research Center (H2020TWINN-2015 (CSA)-692427).

This work is supported by the ERDF European Regional Development Fund through the Operational Programme for Competitiveness and Internationalisation - COMPETE 2020 Programme within project "POCI-01-0145-FEDER-006961", and by National Funds through the Portuguese funding agency, FCT - Fundação para a Ciência e a Tecnologia, as part of project "UID/EEA/50014/2013".

\section{REFERENCES}

[1] R. Campos et al. "BLUECOM+: Cost-effective broadband communications at remote ocean areas". In: OCEANS 2016 - Shanghai. Apr. 2016, pp. 1-6. DOI: 10.1109/OCEANSAP.2016.7485532.

[2] Fang Huang, Xuefei Liao, and Yong Bai. "Multipath Channel Model for Radio Propagation over Sea Surface". In: Wireless Personal Communications 90.1 (2016), pp. 245-257.

[3] Fang Huang, Yong Bai, and Wencai Du. "Maritime radio propagation with the effects of ship motions". In: Journal of Communications 10.5 (2015), pp. 345-351.
[4] S. Siyang and T. Kerdcharoen. "Development of unmanned surface vehicle for smart water quality inspector". In: 2016 13th International Conference on Electrical Engineering/Electronics, Computer, Telecommunications and Information Technology (ECTI-CON). June 2016, pp. 1-5. DOI: 10.1109/ECTICon . 2016. 7561370 .

[5] Nuno Cruz et al. "Zarco-an autonomous craft for underwater surveys". In: 7th Geomatic Week (2007).

[6] Bruno M. Ferreira, Aníbal C. Matos, and José C. Alves. "Water-jet propelled autonomous surface vehicle UCAP: System description and control". In: OCEANS 2016-Shanghai. IEEE. 2016, pp. 1-5.

[7] Anders Lyhne Christensen et al. "Design of Communication and Control for Swarms of Aquatic Surface Drones.” In: ICAART (2). 2015, pp. 548-555.

[8] K. Yang et al. "Channel characterization including path loss and Doppler effects with sea reflections for mobile radio propagation over sea at $2 \mathrm{GHz}$ ". In: 2010 International Conference on Wireless Communications Signal Processing (WCSP). Oct. 2010, pp. 1-6. DOI: 10.1109/WCSP.2010.5633545.

[9] K. Maliatsos et al. "Measurements and Wideband Channel Characterization for Over-the-sea Propagation". In: 2006 IEEE International Conference on Wireless and Mobile Computing, Networking and Communications. June 2006, pp. 237-244. DOI: 10.1109/WIMOB.2006. 1696376.

[10] LEE Yee Hui, Feng Dong, and Yu Song Meng. "Near sea-surface mobile radiowave propagation at $5 \mathrm{GHz}$ : measurements and modeling". In: Radioengineering 23.3 (2014), p. 825.

[11] José Carlos Reyes-Guerrero. "Experimental Broadband Channel Characterization in a Sea Port Environment at $5.8 \mathrm{GHz}$ ". In: IEEE Journal of Oceanic Engineering 41.3 (2016), pp. 509-514.

[12] M. J. Lopes et al. "Wi-Fi broadband maritime communications using $5.8 \mathrm{GHz}$ band". In: 2014 Underwater Communications and Networking (UComms). Sept. 2014, pp. 1-5. DOI: 10.1109/UComms.2014.7017139.

[13] B. Ferreira et al. "Flexible unmanned surface vehicles enabling future internet experimentally-driven research". In: OCEANS 2017 - Aberdeen. June 2017, pp. 1-6. DOI: 10.1109/OCEANSE.2017.8084934.

[14] RW Sinnott. "Virtues of the Haversine". In: Sky and Telescope 68 (1984), p. 158.

[15] Georgia E Athanasiadou. "Incorporating the Fresnel zone theory in ray tracing for propagation modelling of fixed wireless access channels". In: Personal, Indoor and Mobile Radio Communications, 2007. PIMRC 2007. IEEE 18th International Symposium on. IEEE. 2007, pp. 1-5.

[16] Daniel Halperin et al. "802.11 with multiple antennas for dummies". In: ACM SIGCOMM Computer Communication Review 40.1 (2010), pp. 19-25. 